\title{
A branched-chain fatty acid is involved in post-embryonic growth control in parallel to the insulin receptor pathway and its biosynthesis is feedback- regulated in C. elegans
}

\author{
Marina Kniazeva, ${ }^{2}$ Tanya Euler, and Min $\operatorname{Han}^{1}$ \\ Howard Hughes Medical Institute and the Department of Molecular, Cellular, and Developmental Biology, University of \\ Colorado, Boulder, Colorado 80309, USA
}

\begin{abstract}
Growth and development of multicellular organisms are controlled by signaling systems that sense nutrition availability and metabolic status. We report a novel and surprising factor in Caenorhabditis elegans development, the monomethyl branched-chain fatty acid C17ISO, a product of leucine catabolism. We show here that C17ISO is an essential constituent in a novel mechanism that acts in parallel with the food-sensing DAF-2 (insulin receptor)/DAF-16 (FOXO) signaling pathway to promote post-embryonic development, and that the two pathways converge on a common target repressing cell cycle. We show that C17ISO homeostasis is regulated by a SREBP-1c-mediated feedback mechanism that is different from the SREBP-1c-mediated regulation of common fatty acid biosynthesis, as well as by peptide uptake and transport. Our data suggest that C17ISO may act as a chemical/nutritional factor in a mechanism that regulates post-embryonic development in response to the metabolic state of the organism.
\end{abstract}

[Keywords: C17ISO; daf-2; FOXO; elo-5; SREBP; cki-1]

Supplemental material is available at http://www.genesdev.org.

Received May 6, 2008; revised version accepted June 6, 2008.

In Caenborhabditis elegans, environmental cues regulate the decision between two post-embryonic developmental options: reproductive growth and an adaptive larval arrest, known as diapause. There are two distinctive types of larval arrest, L1 (first larval stage) diapause and dauer formation (Johnson et al. 1984; Riddle and Alberts 1997; Muänoz 2003). Dauer larvae, third-stage larvae (dL3) with distinct structures and behaviors suited for long-term survival, are induced at the L1 larval stage by unfavorable growth conditions. The food-sensing insulin/DAF-2 receptor-stimulated pathway that negatively controls activity of a FOXO transcription factor, DAF16 , plays a critical role in dauer formation /Vowels and Thomas 1992; Gottlieb and Ruvkun 1994; Kimura et al. 1997; Lin et al. 1997; Ogg et al. 1997; Gems et al. 1998; Lee et al. 2003). In contrast to dauer formation, the L1 diapause is characterized by a failure to initiate post-

Corresponding authors.

1E-MAIL: mhan@colorado.edu; FAX (303) 735-0175.

${ }^{2}$ E-MAIL: marinak@colorado.edu; FAX (303) 735-0175.

Article is online at http://www.genesdev.org/cgi/doi/10.1101/gad.1692008.

Freely available online through the Genes \& Development Open Access option. embryonic development in the absence of food (Johnson et al. 1984) or at a high temperature (Muänoz 2003).

Fatty acids (FAs) are very diverse in their structures. Whereas the biochemistry and physiology of straight chain FAs have been analyzed extensively using animal models, nearly nothing is known about the homeostasis and functions of branched-chain FAs in animals. In fact, little is known about the roles of any FAs in coordination of animal growth with changes in environment or metabolic status. In the previous study, we described for the first time the essential physiological roles of unusual monomethyl branched-chain FAs (mmBCFA) in C. elegans (Kniazeva et al. 2004). We determined that C. elegans is capable of synthesizing long-chain mmBCFA and showed that the elongation enzyme ELO-5 is required for the biosynthesis of mmBCFAs C17ISO, C15ISO (Kniazeva et al. 2004). Inhibition of the endogenous production of mmBCFAs by elo-5(RNAi) results in various developmental defects in the intestine and gonads, as well as defects in egg laying in the first affected $(\mathrm{PO})$ generation, with progeny $(\mathrm{F} 1)$ arresting at the L1 stage (Kniazeva et al. 2004). Here, we investigate whether C17ISO plays a distinct regulatory role in the 
release from L1 diapause, whether it functions within the food-sensing insulin/DAF-2/DAF-16 pathway reported to play a role in L1 arrest (Baugh and Sternberg 2006; Fukuyama et al. 2006), and how C17ISO homeostasis is regulated. We also speculate on why this particular metabolite may reflect the nutritional balance within the organism.

\section{Results}

C17ISO-deficient animals arrest at the early L1 stage similar to starvation-induced L1 diapause

elo-5 loss-of-function [elo-5(lf)] mutants cultured on plates containing mmBCFA-free bacteria (OP50 plates) fail to grow past the L3 stage, and this defect can be overcome by supplementing with a certain concentration of C17ISO (Supplemental Fig. S1A,B; Kniazeva et al. 2004). When the OP50 plates are supplemented with the same concentration of C13ISO, a likely precursor of C17ISO (Supplemental Material), the F1 animals grow to adults without obvious morphological or developmental defects (Supplemental Fig. S1). However, their progeny (F2) uniformly enter L1 diapause $(n>1000)$ (Fig. 1A), as was described for elo-5(RNAi) animals (Kniazeva et al. 2004). Rescue from the diapause can be achieved by ap-

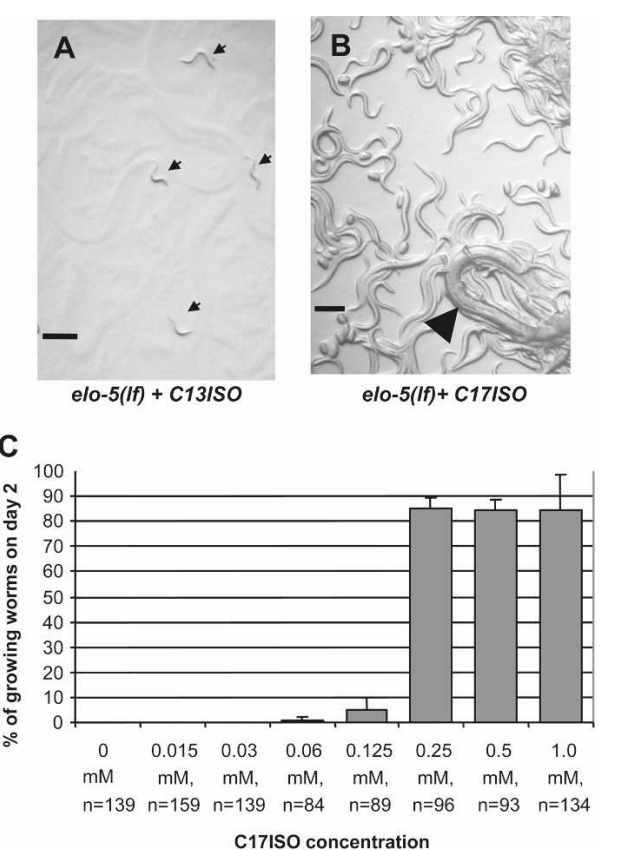

Figure 1. C17ISO is required for post-embryonic development. (A) Progeny of the C17ISO-deficient elo-5(lf) adult uniformly entered L1 diapause (indicated by arrows). (B) The L1-arrested worms were rescued to full growth and propagation after adding $\mathrm{C} 17 \mathrm{ISO}$ to the plates $2 \mathrm{~d}$ after the arrest. Arrowhead points to P0 animal surrounded by its progeny. $(C)$ The C17ISO-depleted elo5(lf) animals were maintained on OP50 plates supplemented with various concentrations of C17ISO from the time of hatching. (n) Number of animals counted in each experiment. Bars, $200 \mu \mathrm{m}$. plying the C17ISO supplement to the top of the bacterial lawn harboring arrested L1s (Fig. 1B; Kniazeva et al. 2004). These results suggest that C17ISO may carry out a critical function required for the initiation of post-embryonic development at L1.

We found that the growth response of the arrested animals to a gradient of supplemental C17ISO concentrations was nonlinear. The nearly all-or-none dosage response is illustrated in Figure 1C. The vast majority of animals that escaped the $\mathrm{L} 1$ arrest developed into normal fertile adults, regardless of the concentration of C17ISO (data not shown), indicating that the amount of C17ISO sufficient to reverse the L1 arrest in this experiment is also sufficient to provide normal reproductive growth.

There are notable similarities between the L1 diapause induced by starvation and the L1 diapause caused by depleting C17ISO: Both occur uniformly at the early L1 stage and both are reversible days after the arrest upon the addition of food and C17ISO, respectively. Using a GFP expressing Escherichia coli OP50-GFP (C. elegans Genetics Center) to trace the food, we determined that the developmental pause induced by C17ISO depletion is not caused by impaired food uptake or starvation (Supplemental Fig. S2).

Moreover, we found that a short-term food deprivation of C17ISO-deficient L1-arrested larvae, followed by supplying C17ISO-free food (OP50), allowed a complete bypass of the C17ISO requirement (data not shown; Materials and Methods). This effect may be due to metabolic or regulatory changes caused by dietary restriction.

We further determined the time of the onset of L1 arrest caused by C17ISO deficiency by examining M-cell divisions. Well-fed wild-type (wt) larva hatch with a single $M$ cell that begins to divide 5-6 h post-hatching, giving rise to 16 cells at the end of L1 (Fig. 2A; Sulston and Horvitz 1977). During L1 diapause caused by starvation, the M cell does not divide (Baugh and Sternberg 2006). Using an hlh-8::GFP reporter expressed in the Mcell lineage (Harfe et al. 1998; Baugh and Sternberg 2006), we did not observed any elo-5(RNAi) larvae with more than one $\mathrm{M}$ cell on the third day of L1 arrest $(n>800)$ (Fig. 2B,C). This result indicates that C17ISO deficiency, like starvation, induces L1 diapause within $5 \mathrm{~h}$ after hatching, even in the presence of food. The stereotypical changes in seam cell shape, highlighted by the AJM-1 protein, which localizes to the dynamic adherens junction, have been shown to correlate with developmental stages during L1 progression (Fig. 2D; Knight et al. 2002). Using an AJM-1 ::GFP reporter transgenic line (Kèoppen et al. 2001), we observed that the seam cell shapes in C17ISO-depleted L1-arrested animals corresponded to a stage $\sim 3-5$ h after hatching (Fig. 2E,F), similar to the ones observed in starved L1-arrested animals.

\section{C17ISO acts in parallel to the DAF-2/}

DAF-16-mediated food-sensing pathway regulating post-embryonic growth and development

The similarity between the C17ISO-related L1 arrest and starvation-induced arrest raised the possibility that 


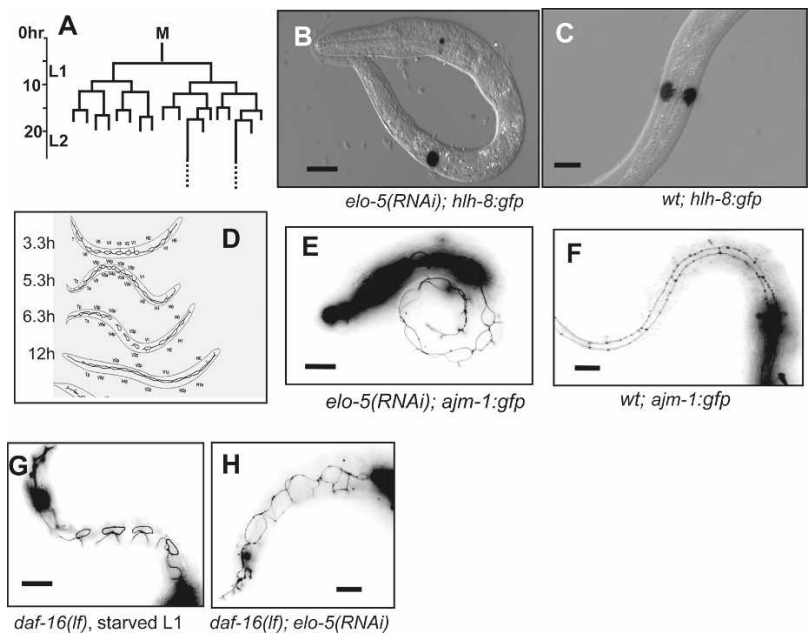

Figure 2. C17ISO deficiency causes developmental arrest in early L1 independently from the DAF-2/DAF-16 food-sensing pathway. (A) The M-cell lineage during L1 development (data adapted from Sulston and Horvitz 1977). (B) hlh-8::GFP expression in a single $\mathrm{M}$ cell in the C17ISO-deficient L1-arrested elo5(RNAi) larva. (C) hlh-8::GFP expression in the two divided $\mathrm{M}$ cells in a wild-type L1 growing larva. $(D)$ The changes in the seam cell patterns during L1 (adopted with permission from Blackwell Publishing [@ 2002; http://www.blackwell-synergy.com] from Knight et al. 2002). (E) AJM-1::GFP expression pattern at adherens junctions in a C17ISO-deficient L1-arrested elo-5(RNAi) larva corresponds to a stage about $3 \mathrm{~h}$ post-hatching (Knight et al. 2002). (F) AJM-1::GFP expression pattern in a wild-type growing $\mathrm{L} 1$ animal corresponding to about $12 \mathrm{~h}$ posthatching. $(G)$ The disconnected seam cell shapes, typical of animals $\sim 6$ h post-hatching $(D)$, were observed in starved daf-16(lf) mutants. $(H)$ The seam cell shapes in daf-16(lf); elo-5(RNAi) are similar to that in elo-5(RNAi) (E), and correspond to a stage about $3.3 \mathrm{~h}$ post-hatching $(D)$.

C17ISO is part of the DAF-2/DAF-16 food-sensing pathway. We first determined that starved L1-arrested animals were not lacking C17ISO. Gas chromatography (GC) analysis of the FA composition in total lipid extracts indicated that C17ISO was present and that the overall FA composition in starved animals was similar to that in well-fed animals (data not shown). We then tested whether C17ISO plays a critical role in activating the DAF-2/DAF-16 signaling pathway. If so, C17ISO added to NGM agar plates lacking bacteria might activate DAF-2 and prevent L1 arrest caused by starvation (Supplemental Fig. S3A). A uniform L1 arrest under this condition was observed (Supplemental Fig. S3B), indicating that C17ISO is not likely to be a major activator of the DAF-2/DAF-16 pathway.

We then tested the possibility that C17ISO is required, but not sufficient for the activation of the DAF-2 pathway at the L1 stage. If C17ISO acts at a step upstream of DAF-16 but downstream from DAF-2 to activate the pathway, a If mutation in daf-18/Pten, which antagonizes the daf-2 activity (Ogg et al. 1997) and/or a If mutation in daf-16, which is negatively regulated by daf-2, may suppress the L1 arrest caused by C17ISO deficiency. However, neither a daf-18(lf) nor a daf-16(lf) was observed to suppress the uniform L1 arrest phenotype caused by elo-5(RNAi) (Supplemental Fig. S4C; data not shown). The inhibition of M-cell proliferation in starved larvae has been shown previously to be associated with DAF-16 activity, as $M$ cells continue to divide in the food-deprived daf-16(lf) mutants (Baugh and Sternberg 2006). A completely penetrant M-cell division arrest was observed in daf-16(RNAi); elo-5(RNAi) animals ( $n>800)$ (data not shown) just as in daf-16(wt);elo-5(RNAi) animals (Fig. 2B), indicating that the inhibition of M-cell division by C17ISO deficiency is independent of DAF-16 activity. Using the AJM-1::GFP reporter, we found that developmental arrest of seam cells in the C17ISO-depleted larvae was also DAF-16 independent, in contrast to the arrest in starved L1 larvae. In starved animals, daf-16(lf) partially released the developmental arrest in the seam cells, as indicated by the AJM-1::GFP marker (Fig. 2G). However, the seam cells in daf-16(lf);elo5(RNAi) animals displayed a similar pattern to that in daf-16(wt); elo-5(RNAi) larvae (Fig. 2, cf. $\mathrm{H}$ and E), corresponding to $\sim 3-5 \mathrm{~h}$ after hatching (Fig. 2D). These data indicate that C17ISO does not act within the DAF-2/ DAF-16 pathway to regulate post-embryonic development. Consistent with this result, the C17ISO content does not change in the daf-2(lf) and daf-16(lf) mutants (data not shown). Additional analysis confirms that the DAF-2/DAF-16 pathway is functional in animals lacking C17ISO (Supplemental Material).

\section{C17ISO acts in a DAF-2/DAF-16-independent pathway to repress cyclin-dependent kinase inhibitor CKI-1}

The similarity in the early onset of the L1 diapauses caused by starvation and by C17ISO deficiency implies that the two mechanisms affect common regulators of post-embryonic growth and development. We evaluated the expression of the cki-1 gene during L1 diapause in C17ISO-deficient animals. cki-1 encodes a cyclin-dependent kinase inhibitor that was shown to be required for cell cycle exit during development and to be strongly expressed in seam cells during L1 arrest caused by starvation (Hong et al. 1998; Baugh and Sternberg 2006). A daf-16-null mutation was shown to significantly reduce the cki-1 expression in seam cells of starvation-induced L1-arrested larvae (Baugh and Sternberg 2006). To examine the expression of cki-1 in C17ISO-deficient larvae, we induced the L1 arrest by feeding elo-5(RNAi) to transgenic animals carrying a cki-1::GFP reporter. While a low level of cki-1::GFP was detected in seam cells in control animals (Fig. 3A,B), a significant increase in the expression was observed in seam cells of the elo5(RNAi)-induced L1-arrested larvae (Fig. 3C). A prominent expression of the reporter was also observed in seam cells in daf-16(1f);elo-5(RNAi) L1-arrested larvae at a level similar to that in the elo-5(RNAi) L1 larvae (Fig. $3 \mathrm{E})$, indicating that the increase in cki-1 expression in C17ISO-deficient L1 animals is daf-16 independent. Based on the above results, we propose that C17ISO plays an essential role in the initiation of post-embryonic growth in parallel to the DAF-2/DAF-16 pathway. 

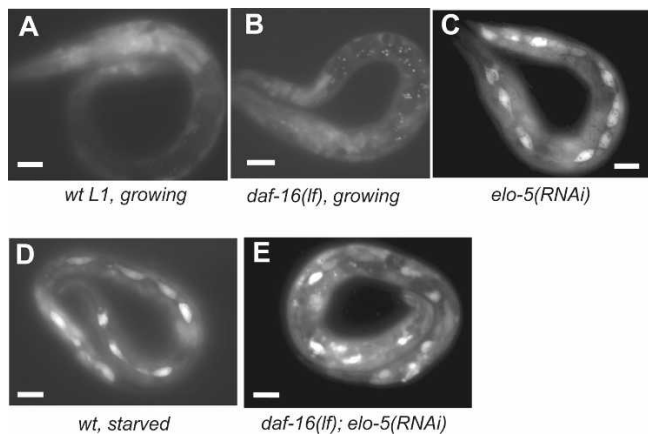

Figure 3. C17ISO acts in parallel to the DAF-2/DAF-16 pathway to repress $c k i-1$ expression in seam cells. $(A-E)$ Images of L1 animals of various genotypes and culture conditions expressing an integrated $c k i-1:: G F P$ reporter in seam cells, as indicated. The animal in $A$ is committed to growth, the animal in $C$ was among progeny of animals that were exposed to elo-5(RNAi) for a lifetime, it is L1-arrested. The GFP expression in seam cells was prominent in L1 animals arrested by deficiency of mmBCFA or by starvation, but not in developing L1, irrelevant to the daf-16 function. Bars, $15 \mu \mathrm{M}$ for all images.

Because the physiological consequences of depleting C17ISO are similar to that of food starvation, the two mechanisms may regulate more common genes in addition to $c k i-1$.

The expression of ACS-1, the enzyme involved in the mmBCFA synthesis, is feedback regulated by C17ISO

The critical role of C17ISO suggests the importance of factors contributing to mmBCFA homeostasis. In a search for genes involved in feedback regulation of mmBCFA biosynthesis, we previously identified acs-1 as a gene significantly up-regulated in elo-5(RNAi) animals deficient in mmBCFAs (Kniazeva et al. 2004). acs-1 encodes a very long-chain acyl-CoA synthetase that plays an essential role in mmBCFA biosynthesis and activation (Supplemental Material; Kniazeva et al. 2004). An acs-1Prom::GFP reporter is expressed in intestinal cells and canal-associated neurons (CAN) in L4 and adult animals (Fig. 4A; Kniazeva et al. 2004). We found that the GFP expression from this reporter was drastically increased in acs-1(RNAi) animals maintained with the C13ISO supplement (and therefore deficient for C17ISO) (Fig. 4B). In contrast, the increase in GFP expression was absent in the acs-1(RNAi) worms maintained with C17ISO supplementation (Fig. 4C), which were visually indistinguishable in growth and morphology from those supplemented with C13ISO. Thus, C17ISO, not C13ISO, negatively regulates acs-1 expression.

\section{SREBP-1c homolog SBP-1 mediates the regulation} of acs-1 expression by C17ISO

We then tested whether sbp-1, which encodes the SREBP-1c homolog in C. elegans (McKay et al. 2003), and nhr-49, encoding a nuclear hormone receptor, play roles in C17ISO-mediated regulation of acs-1 expression. Both transcription factors were selected from our screen for genes potentially sensitive to mmBCFA deficiency (Kniazeva et al. 2004). sbp-1 has been shown to regulate mmBCFA biosynthesis as well as the expressions of elo-5 and elo-6 (Kniazeva et al. 2004), while nhr-49 has been shown to play a role in straight-chain FA homeostasis (Kniazeva et al. 2004; Van Gilst et al. 2005a,b). In acs-1(RNAi) animals supplemented with C13ISO, a suppression of $s b p-1$, but not $n h r-49$, by RNAi eliminated the stimulating effect of C17ISO deficiency on acs1Prom:: GFP expression (Fig. 4D,E).

We further used real-time RT-PCR (qPCR) to quanti-

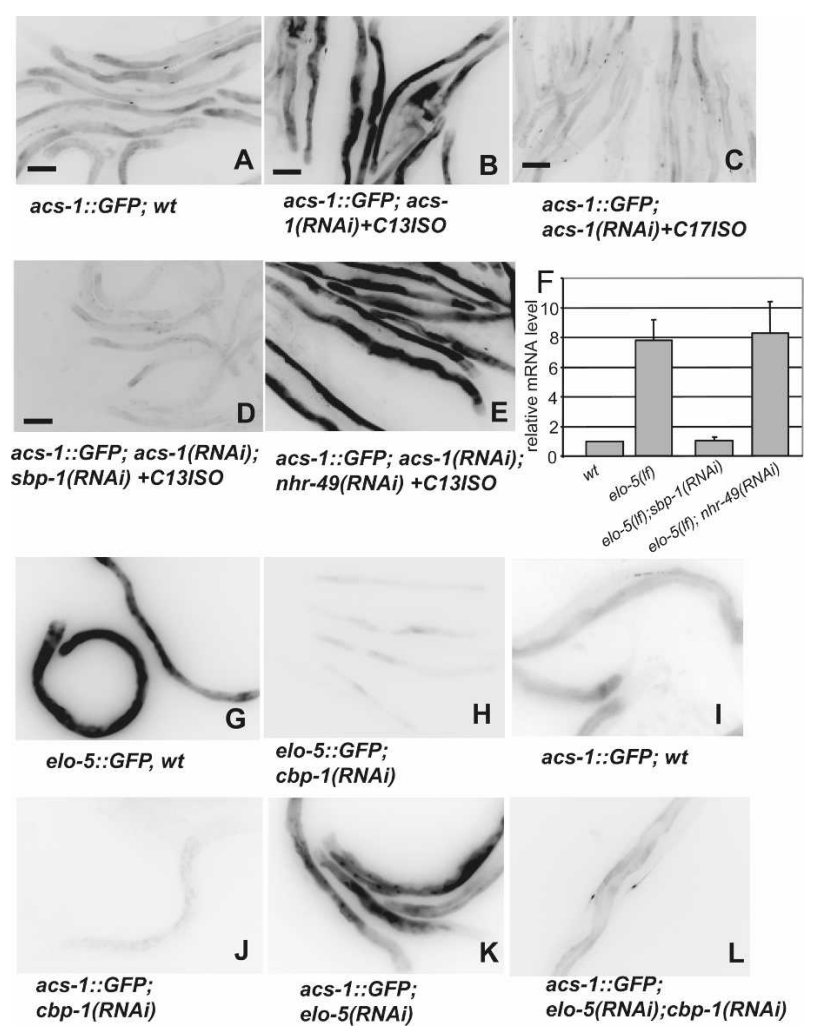

Figure 4. acs-1 expression is up-regulated by deficiency of $\mathrm{C} 17 \mathrm{ISO}$ but not $\mathrm{C} 13 \mathrm{ISO}$, and this regulation requires transcription factors $s b p-1$ and $c b p-1$. $(A-E)$ Images of worms expressing GFP from the acs-1Prom::GFP reporter transgene. RNAi treatment and mmBCFA supplementations are indicated. A moderate expression of the reporter in a wild-type background $(A)$ increased dramatically following the depletion of C17ISO [acs1(RNAi) + C13ISO] $(B) .(C)$ The increase was abolished in the experiments where C13ISO supplementation was replaced with C17ISO. RNAi of $s b p-1(D)$, but not $n h r-49(E)$, inhibited the acs-1 up-regulation. Bars, $100 \mu \mathrm{M}$. $(F)$ Results of a quantitative RT-PCR analysis of the endogenous acs-1 gene expression in C17ISO-deficient animals. The levels are relative to a wild-type sample. $(G-L)$ Images showing the GFP expression from either the elo-5Prom::GFP or the acs-1Prom::GFP transgene in wildtype or RNAi-treated animals as indicated. RNAi of $c b p$-1inhibits the steady state elo-5 expression as well as the up-regulation of acs-1 by mmBCFA deficiency. 
tatively measure the mRNA level of the endogenous acs-1 gene under the tested conditions. C17ISO-deficient animals were obtained by culturing elo-5(lf) mutants in the presence of C13ISO supplementation. The result indicates that the deficiency of C17ISO, but not C13ISO, caused an approximate sevenfold increase in the acs-1 mRNA level and the increase was dependent on $s b p-1$ but not on nhr-49 (Fig. 4F). These results indicate that the end product of mmBCFA biosynthesis, C17ISO, negatively regulates acs-1 expression through modulating SBP-1 activity.

\section{CBP-1, but not MDT-15, is involved in SBP-1} mediated regulation of $\mathrm{mmBCFA}$ biosynthesis

In mammals and C. elegans a transcription mediator, ARC105 (worm homolog MDT-15), cooperates with SREBP-1c (SBP-1) for their functions in FA synthesis (Taubert et al. 2006; Yang et al. 2006). We found MDT-15 is unlikely involved in mmBCFA biosynthesis, as no significant changes in levels of branched-chain FA were detected in mdt-15(RNAi) animals (Fig. 5A,B). We also tested $c b p-1$, which encodes the $C$. elegans homolog of mammalian CBP/p300, another cofactor capable of binding to SREBP-1c in mammals (Yang et al. 2006). Previous reports showed that CBP-1 is a critical transcriptional cofactor in promoting cell differentiations (Shi and Mello 1998; Eastburn and Han 2005), but its role in FA metabolism has not been analyzed. We found that elo5Prom::GFP expression was down-regulated and the response of acs-1Prom::GFP expression to the C17ISO deficiency was abolished in $c b p-1$ (RNAi) animals (Fig. 4GJ; Supplemental Fig. S7C,D). cbp-1(RNAi) animals were also severely deficient in mmBCFAs as well as in polyunsaturated 20-carbon straight-chain FAs (C20 PUFA) (Fig. 5C). These $c b p-1$ (RNAi) effects on mmBCFA production were similar to that of $s b p-1$ (RNAi) (Fig. 4), suggesting that CBP-1, but not MDT-15, collaborates with SBP-1 in regulating mmBCFA biosynthesis.

\section{$m m B C F A$ biosynthesis is regulated by protein uptake} and transport

Since leucine is a precursor of C17ISO, we investigated additional links between protein and mmBCFA homeostasis. pep-2 encodes an essential oligopeptide transporter, which is involved in absorption of digested proteins by intestinal cells in C. elegans (Supplemental Fig. S7E,F; Nehrke 2003; Meissner et al. 2004). GC analysis showed that pep-2(RNAi) caused a drastic reduction of mmBCFAs, along with a significant reduction in the levels of polyunsaturated FAs (PUFAs) (Fig. 5D). Since supplementing pep-2(RNAi) animals with C13ISO did not significantly restore C17ISO levels (data not shown), we examined the expression of GFP driven by promoters of mmBCFA biosynthetic genes, elo-5, acs-1, and $s b p-1$ in pep-2(RNAi) animals (Fig. 5E-J). A decrease in the expression of elo-5Prom::GFP was observed (Fig. 5E,F). Therefore, transcriptional down-regulation of elo-5 may
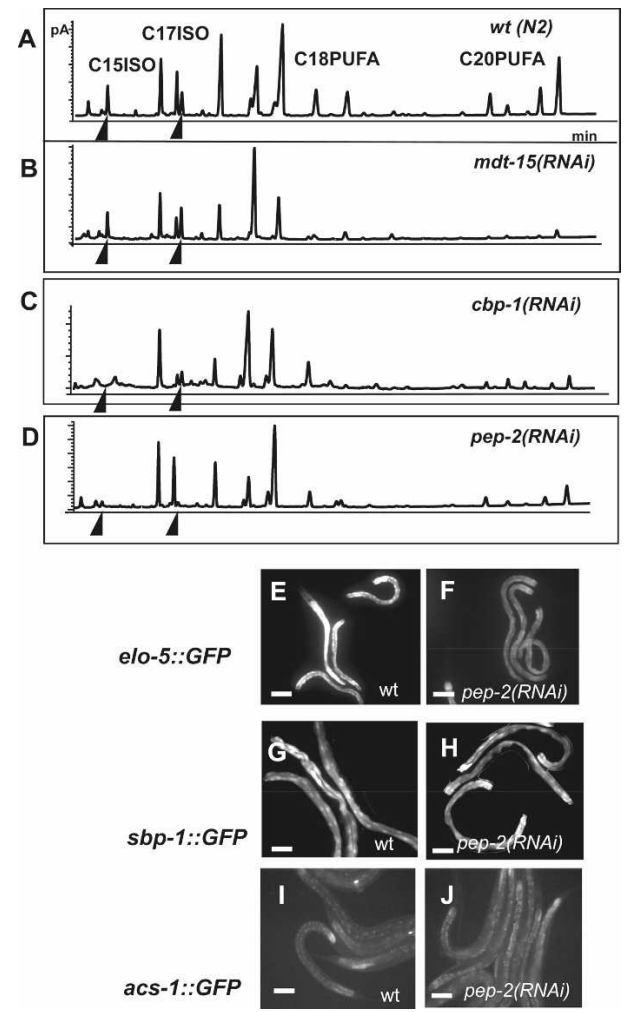

Figure 5. pep-2(RNAi) and $c b p-1(\mathrm{RNAi})$, but not $m d t$ 15(RNAi), significantly influence mmBCFA biosynthesis. $(A-D)$ GC analysis of FA content in total lipid extracts obtained from RNAi-treated strains as indicated. Arrowheads point to the C15ISO and C17ISO peaks. Drastic decreases in mmBCFA biosynthesis were observed in animals treated with pep-2(RNAi) and $c b p-1(R N A i)$, but not animals with $m d t-15$ (RNAi). By comparison, all three RNAi treatments affected the fractions of C20 PUFA. $(E-J)$. GFP images showing the expression of the elo5Promoter::GFP or sbp-1Promoter::GFP or acs1Promoter::GFP transgene in the wild-type or pep-2(RNAi) strain as indicated. pep-2(RNAi) causes a significant reduction of the expression of elo-5:: GFP but not the expression of $s b p-1:: G F P$ or asc1::GFP. Bars, $100 \mu \mathrm{M}$.

at least in part be responsible for mmBCFA deficiency in pep-2(RNAi). It has recently been shown that leucine deprivation in mammals causes down-regulation of genes related to the FA biosynthesis by reducing mRNA and protein levels of SREBP (Guo and Cavener 2007). Thus, it is possible that impaired peptide transport may mimic essential amino acid deficiency and may affect elo-5 transcription through regulating $s b p-1$, although our GFP reporter analysis suggests that this potential regulation on $s b p-1$ would be more likely through a posttranscriptional mechanism (Fig. 5G,H). The fact that wild-type animals left without food for $2 \mathrm{~d}$ have an essentially normal mmBCFA composition (data not shown) suggests that it is not a deficiency of dietary amino acids per se, but the imbalance in these metabolites that is associated with the dramatic down-regulation of mmBCFA. It implies that levels of mmBCFAs largely depend on coordinated lipid and protein homeostasis, rather than on nutrient deprivation. 


\section{Discussion}

C17ISO is an essential constituent of a mechanism regulating initiation of post-embryonic development in C. elegans

C. elegans hatched with C17ISO deficiency enter a diapause at the first larval stage (L1), which resembles the L1 arrest in a food-free environment. The diapause is uniform and occurs at the same developmental stage at which the starved L1 larvae are arrested. The diapause is reversible when C17ISO is supplied, as is the L1 arrest caused by starvation with the presence of food (Johnson et al. 1984; Gems et al. 1998). Furthermore, as in starved L1-arrested animals, an inhibitor of cell cycle, cki-1, is up-regulated during L1 diapause in C17ISO-deficient larvae

The previous observations suggest that starvation induces L1 arrest through the insulin/DAF-2/DAF-16 signaling pathway (Gems et al. 1998; Pierce et al. 2001; Baugh and Sternberg 2006; Fukuyama et al. 2006; Kao et al. 2007). We showed that C17ISO functions in a mechanism that is independent of the DAF-2/DAF-16 signaling cascade. The signaling activity through the DAF-2/DAF16 pathway is neither sufficient to mediate the C17ISO function, nor necessary for the L1 arrest, including cell cycle arrest in $\mathrm{M}$ and seam cell lineages caused by C17ISO deficiency. Thus, C17ISO is necessary to suppress a negative regulator of the cell cycle independently from food-stimulated insulin/DAF-2/DAF-16 pathway.

These results raise further questions about the intriguing relationship between these two independently operating insulin/DAF-2/DAF-16 and C17ISO-responsive regulatory mechanisms. In the regulation of $c k i-1$ expression, the C17ISO deficiency induces cki-1::GFP expression that is epistatic to the effect of a loss-of-function mutation in daf-16. It is conceivable that in wildtype L1 animals hatched in the presence of food, C17ISO mediates a repression of $c k i-1$, whereas an activated DAF-16 acts to relieve this repression upon starvation. Given a similarity between L1 arrests caused by C17ISO deficiency and starvation, it is likely that cki-1 is not the only target common between DAF-2/DAF-16 and C17ISO-responsive mechanisms.

\section{C17ISO homeostasis is maintained through} a feedback transcriptional regulation mediated by $S B P-1$

The homeostasis of C17ISO in adult animals appears to be strictly regulated. We found that the expression of one of the essential mmBCFA biosynthetic genes, acs-1, is significantly up-regulated by the deficiency of C17ISO and this up-regulation depends on $s b p-1$ (Fig. 4). It was shown previously that SBP-1 utilizes MDT-15 as an essential cofactor in its regulation of straight-chain FA biosynthesis (McKay et al. 2003; Taubert et al. 2006 ; Yang et al. 2006). The fact that $m d t-15$ is not required for mmBCFA production may suggest that $s b p-1$-mediated feedback regulation of acs-1 expression depends on another, possibly mmBCFA-specific cofactor, and that the regulation of mmBCFA biosynthesis by SBP-1 may be uncoupled from a regulation of general lipid biosynthesis through this hypothetical mmBCFA-specific cofactor.

\section{C17ISO level may sense multiple essential molecules and nutrients}

It would be reasonable to suggest that L1 larvae sense many different metabolites before deciding whether to develop or arrest, although a potential mechanism of metabolite-dependent growth control is totally unknown. Since mmBCFAs are required for the genesis and maintenance of structure and morphology in C. elegans at all developmental stages (Kniazeva et al. 2004), the evaluation of the mmBCFA levels at early L1 could be beneficial for survival. Our data show that mmBCFA biosynthesis is tightly linked to the overall lipid metabolism through the regulation by SBP-1, to the protein metabolism through an essential amino acid, leucine, a precursor of mmBCFA, and through amino acid/peptide uptake and transport mediated by PEP-2 (Fig. 6). Since C17ISO is the final product of consecutive steps of mmBCFA biosynthesis, its levels intrinsically "sense" mmBCFA production and may reflect the levels of essential amino acids, and, more generally, protein and lipid homeostasis of the organism (Fig. 6).

In conclusion, C17ISO is an essential metabolite and its presence is required, in addition to the insulin-activated DAF-2/DAF-16 pathway, for the L1 larvae to release from the cell cycle arrest. The studies on C17ISO will be important in understanding the metabolite-de-

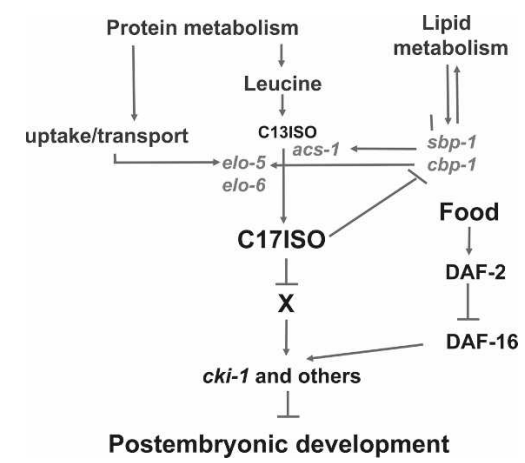

Figure 6. A model for a regulatory function and homeostasis of a long-chain mmBCFA in C. elegans. Arrows and T bars illustrate positive and negative interactions, respectively. The data provided in this study suggest that $C$. elegans has a novel regulatory mechanism that involves mmBCFA to control the onset of post-embryonic growth and development. C17ISO is required to repress cki-1 and other growth regulators through the interaction with an unknown factor(s) (X). This mechanism functions in parallel to the well-known insulin receptor pathway that is capable of sensing food (Gems et al. 1998). The diagram also indicates a feedback regulatory circuit that plays a critical role in C17ISO homeostasis. C17ISO may represent critical metabolic and growth conditions that include the levels of mmBCFA and essential amino acids, protein uptake and transport, and certain lipid metabolic conditions. 
pendent growth control and the physiological roles of branched chain FAs in animals, including humans.

\section{Materials and methods}

\section{Worm strains and assays in general}

The wild-type strain was N2 Bristol. A deletion strain elo5(gk208) was obtained from the C. elegans knockout consortium. The following alleles including transgenic genes were obtained from the Caenorhabditis Genetic Center or from the laboratory indicated in the reference: hlh-8::GFP(ayIs7 IV), ajm-1::GFP (jcIs1) IV, cki-1::GFP, daf-2(e1370)III, daf16(mgDf50) I, daf-18(nr2037) (Gil et al. 1999), and maIs113 (cki$1:: G F P$ ) (Baugh and Sternberg 2006), and sbp-1::GFP (McKay et al. 2003). elo-5(gk208), acs-1Prom::GFP, elo-5Prom::GFP, and elo-6Prom::GFP were described previously (Kniazeva et al. 2004).

Unless otherwise specified, C. elegans strains were maintained under standard conditions on NGM agar with Escherichia coli OP50 as a food source at $20^{\circ} \mathrm{C}$. The daf-2(e1370) animals carry a reduction-of-function (rf), or partial loss-of-function (lf) allele and become dauers at $20^{\circ} \mathrm{C}$. To prevent the dauer formation, parental worms were initially maintained at the permissive temperature, $15^{\circ} \mathrm{C}$, and switched to $20^{\circ} \mathrm{C}$ at the $\mathrm{L} 4$ or young adult stage to inactivate the gene. All experiments and assays started from plating eggs released from parental animals by bleaching onto corresponding plates. Unless specified differently, all assays were done at least three times and a set of three to five plates containing 100-150 animals were analyzed for each condition tested.

\section{FA supplements}

C13ISO, C15ISO, or C17ISO (Sigma, Matreya, or Larodan) were dissolved in $100 \%$ DMSO at $10 \mathrm{mM}$ concentration and mixed with overnight bacterial culture to $1 \mathrm{mM}$ final concentration prior to spotting plates. The bacteria/supplements were spotted onto NGM agar plates. The plates were left to dry with the lids on. Control plates were prepared by mixing DMSO into overnight bacterial suspension to a final concentration of $10 \%$ prior to spotting plates. Experimental and control plates for a given assay were prepared with a single source of FA- or DMSOsupplemented bacterial food.

\section{Maintenance of the elo-5(lf) homozygous mutant}

The homozygous lethal elo-5(lf) strain is maintained on NGM plates seeded with E. coli OP50 mixed with supplementary 1 $\mathrm{mM}$ C17ISO dissolved in $10 \%$ DMSO (final concentration in bacterial suspension) or on NGM plates seeded with a mixture of OP50 and mmBCFA-producing bacteria Stenotrophomonas maltophilia.

\section{Generation of mmBCFA-depleted animals}

elo-5(lf) adults maintained on S. maltophilia were bleached and the released eggs were placed on E. coli OP50 or HT115 seeded plates. Alternatively, wild-type or mutant strains were subjected to elo-5(RNAi) or to acs-1(RNAi) without mmBCFA supplements. "HT115" refers to E. coli strain HT115 transformed with the empty pPD129.36 RNAi vector.

\section{Generation of C17ISO-depleted adults}

elo-5(lf) adults maintained on S. maltophilia were bleached and the released eggs were placed on E. coli OP50 or HT115 seeded plates supplemented with C13ISO. Worms of the first generation (P0) displayed superficially normal growth and development.

\section{Generation of C17ISO-depleted L1 arrested animals}

elo-5 (gk208; If) adults (P0) maintained on S. maltophilia or C17ISO supplements were bleached and the released eggs (F1) were placed on OP50 or HT115 plates supplemented with C13ISO. Three days after plating, adult F1s were bleached to release eggs (F2) on the plates according to experimental tasks. Without C17ISO supplements, the eggs (F2) gave rise to the C17ISO-depleted L1-arrested larvae.

To generate C17ISO-depeleted L1 arrest larvae in other mutant backgrounds, P0 worms were subjected to elo-5(RNAi) without any mmBCFA supplements from the time of hatching. This treatment resulted in gradual mmBCFA depletion in the P0 generation. The adults were maintained on the same plates or bleached, and eggs (F1) were plated onto fresh plates prepared according to the experimental tasks. The resulting F1 generation was C17ISO-depleted and L1-arrested.

\section{L1 arrest reversal through food deprivation}

The C17ISO-depleted L1-arrested animals were obtained from the elo-5(gk208) parents as indicated above. The larvae were cultured on food-free NGM plates supplemented with DMSO, C13ISO, or nothing for $2 \mathrm{~d}$. Afterward, a small amount of OP50 paste was added to all the conditions. Animals were evaluated the next day for growth using dissection and high-resolution light microscopy. Lipid extracts were prepared from propagating animals and the FA composition was analyzed by GC. The result indicated that these starvation-rescued animals did not produce more C17ISO (data not shown). The elo-5(lf) genotype of the rescued animals was verified by PCR.

\section{Strains and conditions for RNAi treatment}

RNAi feeding strains used in this study were obtained from the C. elegans whole-genome RNAi feeding library (J. Ahringer, MRC Geneservice). The RNAi feeding control strain was E. coli HT115 transformed with the empty pPD129.36 vector. All RNAi strains used were verified for the correct inserts by sequencing the isolated plasmids. RNAi plates were prepared as described in Kamath et al. (2001). When RNAi plates included mmBCFA supplements, two controls were used: (1) plates with the RNAi strain alone and (2) plates with the same RNAi strain supplemented with $10 \%$ DMSO.

\section{Analysis of growth response to concentrations of C17ISO}

Worms maintained on $S$. maltophilia plates were collected, rinsed in water, and bleached. The eggs were plated onto OP50 plates supplemented with $1 \mathrm{mM}$ C13ISO in DMSO. The hatched animals grew to adults depleted of C17ISO. On day 3 post-hatching, adults were collected, rinsed with water, and the eggs were plated on OP50 plates supplemented with different concentrations of C17ISO. The eggs were placed outside a bacterial lawn so that $\mathrm{L} 1$ animals that were damaged or ill from the treatment, and thus unable to move toward the food, were not scored. The animals were observed for the following $4 \mathrm{~d}$, during which the structure and developmental stages were recorded. Aliquots of C17ISO in DMSO $(50 \mu \mathrm{L})$ were mixed with $450 \mu \mathrm{L}$ of OP50 bacteria before spotting plates to obtain final concentrations of $0.015,0.03,0.06,0.125,0.25,0.5$, and $1.0 \mathrm{mM}$. (Note: The actual concentration of C17ISO on a plate can vary due to 
the limited solubility of C17ISO.) Error bars reflect variations within three biological replicates used in the experiment.

\section{GFP expression analysis}

GFP fluorescence was observed using a Zeiss Axioplan microscope. The images were captured with a Hamamatsu ORCA-ER digital camera.

Animals were anesthetized on agar pads in a drop of $10 \mathrm{mM}$ $(0.7 \%)$ sodium azide (Sulston and Hodgkin 1988) and analyzed immediately. Specimens were scored within 5 min after preparing the slides. For comparative analysis of GFP expression, experimental and control animals were mounted on the same slide. GFP images were obtained with the fixed settings.

\section{Developmental stage and morphology evaluation}

Growth and development was monitored using a Leica MZ6 dissecting scope equipped with a Canon PowerShot A620 digital camera, and using Nomarski optics on a Zeiss Axioplan microscope.

\section{GC analysis}

Worms were washed off the plates with water, rinsed three to four times, and the pellet was frozen at $-80^{\circ} \mathrm{C}$. Lipid extraction and FA methyl esters were prepared as described (Miquel and Browse 1992). GC was performed on a HP6890N (Agilent) apparatus equipped with a DB-23 column $(30 \mathrm{~m} \times 250 \mu \mathrm{m} \times 0.25$ $\mu \mathrm{m})$ from Agilent. Each experiment was repeated at least five times.

\section{Quantitative RT-PCR}

The C17ISO-depleted adults were obtained from the elo-5(lf) parents, maintained as described above. The eggs were released by bleaching and plated on HT115, sbp1-(RNAi), and nhr49(RNAi) supplemented with C13ISO. Wild-type animals maintained on HT115 supplemented with C13ISO from the time of hatching were used as a normal reference. Three days after plating the eggs, samples of total RNA were prepared from corresponding mixed populations of adults, eggs, and L1-arrested larvae according to the RNeazy protocol (Qiagen). q-PCR was done according to SYBR-protocol (Sigma). rpl-21 mRNA was used as an internal reference. Three biological and six technical replicates were used for each sample. Average and standard deviation were calculated in Excel. Relative quantification analysis was performed using the $2^{-\Delta \Delta C T}$ Method (Livak et al. 2001).

\section{Acknowledgments}

We thank Jon Graff for the 1pd-1::GFP strain; Paul Sternberg for daf-16(mgDf50); maIs113 (cki-1::GFP); Yo Suzuki for the daf16(mgDf50);ajm-1::GFP; Andy Fire for vectors; and Aileen Sewell, Jennifer Blanchette, Emylie Seamen, Chen Wang, Bill Wood, and members of our laboratory for valuable discussions during the study and comments on the manuscript. This project is supported by the Howard Hughes Medical Institute, of which M.K., T.E., and M.H. are a research specialist, a research technician, and an investigator, respectively.

\section{References}

Baugh, L.R. and Sternberg, P.W. 2006. DAF-16/FOXO regulates transcription of cki-1/Cip/Kip and repression of lin-4 during C. elegans L1 arrest. Curr. Biol. 16: 780-785.
Eastburn, D.J. and Han, M. 2005. A gain-of-function allele of cbp-1, the Caenorhabditis elegans ortholog of the mammalian CBP/p300 gene, causes an increase in histone acetyltransferase activity and antagonism of activated Ras. Mol. Cell. Biol. 25: 9427-9434.

Fukuyama, M., Rougvie, A.E., and Rothman, J.H. 2006. C. elegans DAF-18/PTEN mediates nutrient-dependent arrest of cell cycle and growth in the germline. Curr. Biol. 16: 773779 .

Gems, D., Sutton, A.J., Sundermeyer, M.L., Albert, P.S., King, K.V., Edgley, M.L., Larsen, P.L., and Riddle, D.L. 1998. Two pleiotropic classes of daf-2 mutation affect larval arrest, adult behavior, reproduction and longevity in Caenorhabditis elegans. Genetics 150: 129-155.

Gil, E.B., Malone Link, E., Liu, L.X., Johnson, C.D., and Lees, J.A. 1999. Regulation of the insulin-like developmental pathway of Caenorhabditis elegans by a homolog of the PTEN tumor suppressor gene. Proc. Nat1. Acad. Sci. 96: 2925-2930.

Gottlieb, S. and Ruvkun, G. 1994. daf-2, daf-16 and daf-23: Genetically interacting genes controlling Dauer formation in Caenorhabditis elegans. Genetics 137: 107-120.

Guo, F. and Cavener, D.R. 2007. The GCN2 eIF2 $\alpha$ kinase regulates fatty-acid homeostasis in the liver during deprivation of an essential amino acid. Cell Metab. 5: 103-114.

Harfe, B.D., Vaz Gomes, A., Kenyon, C., Liu, J., Krause, M., and Fire, A. 1998. Analysis of a Caenorhabditis elegans Twist homolog identifies conserved and divergent aspects of mesodermal patterning. Genes \& Dev. 12: 2623-2635.

Hong, Y., Roy, R., and Ambros, V. 1998. Developmental regulation of a cyclin-dependent kinase inhibitor controls postembryonic cell cycle progression in Caenorhabditis elegans. Development 125: 3585-3597.

Johnson, T.E., Mitchell, D.H., Kline, S., Kemal, R., and Foy, J. 1984. Arresting development arrests aging in the nematode Caenorhabditis elegans. Mech. Ageing Dev. 28: 23-40.

Kamath, R.S., Martinez-Campos, M., Zipperlen, P., Fraser, A.G., and Ahringer, J. 2001. Effectiveness of specific RNA-mediated interference through ingested double-stranded RNA in Caenorhabditis elegans. Genome Biol. 2: research0002.1research0002.10. doi: 10.1186/gb-2000-2-1-research0002.

Kao, G., Nordenson, C., Still, M., Rèonnlund, A., Tuck, S., and Naredi, P. 2007. ASNA-1 positively regulates insulin secretion in C. elegans and mammalian cells. Cell 128: 577-587.

Kèoppen, M., Simske, J.S., Sims, P.A., Firestein, B.L., Hall, D.H., Radice, A.D., Rongo, C., and Hardin, J.D. 2001. Cooperative regulation of AJM-1 controls junctional integrity in Caenorhabditis elegans epithelia. Nat. Cell Biol. 3: 983-991.

Kimura, K.D., Tissenbaum, H.A., Liu, Y., and Ruvkun, G. 1997. daf-2, an insulin receptor-like gene that regulates longevity and diapause in Caenorhabditis elegans. Science 277: 942946.

Kniazeva, M., Crawford, Q.T., Seiber, M., Wang, C.Y., and Han, M. 2004. Monomethyl branched-chain fatty acids play an essential role in Caenorhabditis elegans development. PLoS Biol. 2: E257. doi: 10.1371/journal.pbio.0020257.

Knight, C.G., Patel, M.N., Azevedo, R.B., and Leroi, A.M. 2002. A novel mode of ecdysozoan growth in Caenorhabditis elegans. Evol. Dev. 4: 16-27.

Lee, S.S., Kennedy, S., Tolonen, A.C., and Ruvkun, G. 2003. DAF-16 target genes that control C. elegans life-span and metabolism. Science 300: 644-647.

Lin, K., Dorman, J.B., Rodan, A., and Kenyon, C. 1997. daf-16: An HNF-3/forkhead family member that can function to double the life-span of Caenorhabditis elegans. Science 278: 1319-1322.

Livak, K.J., Schmittgen, T.D., and Applied Biosystems, 
F.C.C.U.S.A. 2001. Analysis of relative gene expression data using real-time quantitative PCR and the $2^{-\Delta \Delta C(T)}$ Method. Methods 25: 402-408.

McKay, R.M., McKay, J.P., Avery, L., and Graff, J.M. 2003. C elegans: A model for exploring the genetics of fat storage. Dev. Cell 4: 131-142.

Meissner, B., Boll, M., Daniel, H., and Baumeister, R. 2004. Deletion of the intestinal peptide transporter affects insulin and TOR signaling in Caenorhabditis elegans. J. Biol. Chem. 279: 36739-36745.

Miquel, M. and Browse, J. 1992. Arabidopsis mutants deficient in polyunsaturated fatty acid synthesis. Biochemical and genetic characterization of a plant oleoyl- phosphatidylcholine desaturase. J. Biol. Chem. 267: 1502-1509.

Muänoz, M.J. 2003. Longevity and heat stress regulation in Caenorhabditis elegans. Mech. Ageing Dev. 124: 43-48.

Nehrke, K. 2003. A reduction in intestinal cell pHi due to loss of the Caenorhabditis elegans $\mathrm{Na}^{+} / \mathrm{H}^{+}$exchanger NHX-2 increases life span. J. Biol. Chem. 278: 44657-44666.

Ogg, S., Paradis, S., Gottlieb, S., Patterson, G.I., Lee, L., Tissenbaum, H.A., and Ruvkun, G. 1997. The Fork head transcription factor DAF-16 transduces insulin-like metabolic and longevity signals in C. elegans. Nature 389: 994-999.

Pierce, S.B., Costa, M., Wisotzkey, R., Devadhar, S., Homburger, S.A., Buchman, A.R., Ferguson, K.C., Heller, J., Platt, D.M., Pasquinelli, A.A., et al. 2001. Regulation of DAF-2 receptor signaling by human insulin and ins-1, a member of the unusually large and diverse C. elegans insulin gene family. Genes \& Dev. 15: 672-686.

Riddle, D.L. and Alberts, P.S. 1997. Genetic and environmental regulation of dauer larva development. In C. elegans $I I$ (eds. D.L. Riddle et al.), pp. 739-768. Cold Spring Harbor Laboratory Press, Cold Spring Harbor, NY.

Shi, Y. and Mello, C. 1998. A CBP/p300 homolog specifies multiple differentiation pathways in Caenorhabditis elegans. Genes \& Dev. 12: 943-955.

Sulston, J.E. and Hodgkin, J. 1988. Methods. In The nematode Caenorhabditis elegans (eds. W.B. Wood et al.), pp. 587-606. Cold Spring Harbor Laboratory Press, Cold Spring Harbor, NY.

Sulston, J.E. and Horvitz, H.R. 1977. Post-embryonic cell lineages of the nematode, Caenorhabditis elegans. Dev. Biol. 56: $110-156$

Taubert, S., Van Gilst, M.R., Hansen, M., and Yamamoto, K.R. 2006. A Mediator subunit, MDT-15, integrates regulation of fatty acid metabolism by NHR-49-dependent and -independent pathways in C. elegans. Genes \& Dev. 20: 1137-1149.

Van Gilst, M.R., Hadjivassiliou, H., Jolly, A., and Yamamoto K.R. 2005a. Nuclear hormone receptor NHR-49 controls fat consumption and fatty acid composition in C. elegans. PLoS Biol. 3: e53. doi: 10.1371/journal.pbio.0030053.

Van Gilst, M.R., Hadjivassiliou, H., and Yamamoto, K.R. 2005b. A Caenorhabditis elegans nutrient response system partially dependent on nuclear receptor NHR-49. Proc. Nat1. Acad. Sci. 102: 13496-13501.

Vowels, J.J. and Thomas, J.H. 1992. Genetic analysis of chemosensory control of dauer formation in Caenorhabditis elegans. Genetics 130: 105-123.

Yang, F., Vought, B.W., Satterlee, J.S., Walker, A.K., Jim Sun, Z.Y., Watts, J.L., Debeaumont, R., Mako Saito, R., Hyberts, S.G., Yang, S., et al. 2006. An ARC/Mediator subunit required for SREBP control of cholesterol and lipid homeostasis. Nature 442: 700-704. 


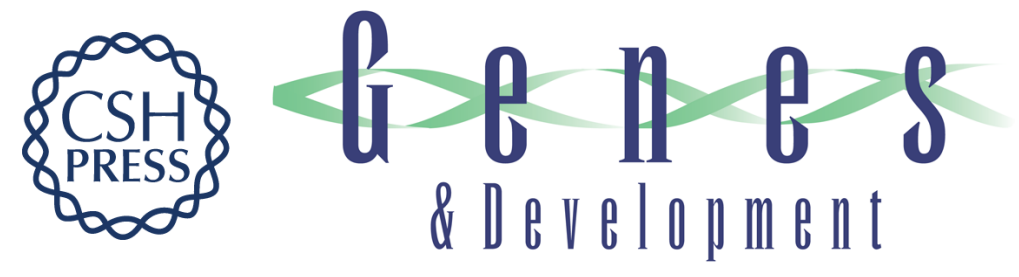

\section{A branched-chain fatty acid is involved in post-embryonic growth control in parallel to the insulin receptor pathway and its biosynthesis is feedback-regulated in C. elegans}

Marina Kniazeva, Tanya Euler and Min Han

Genes Dev. 2008, 22:

Access the most recent version at doi:10.1101/gad.1692008

Supplemental
Material http://genesdev.cshlp.org/content/suppl/2008/08/05/22.15.2102.DC1

Related Content Fatty Acid-Regulated Diapause

Annalisa M. VanHook

Sci. Signal. August, 2008 1: ec289

References This article cites 33 articles, 17 of which can be accessed free at:

http://genesdev.cshlp.org/content/22/15/2102.full.html\#ref-list-1

Articles cited in:

http://genesdev.cshlp.org/content/22/15/2102.full.html\#related-urls

License Freely available online through the Genes \& Development Open Access option.

Email Alerting Receive free email alerts when new articles cite this article - sign up in the box at the top Service right corner of the article or click here.

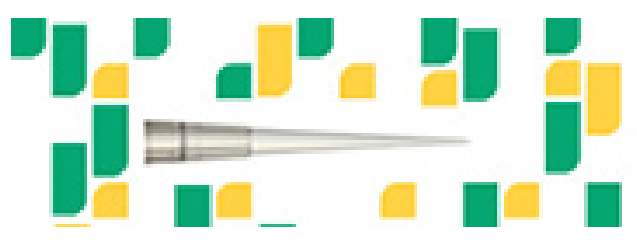

Focused on your science. 